\title{
Wechselwirkung Mensch und autonomer Agent
}

\author{
Ingo Wolf
}

\section{Inhaltsverzeichnis}

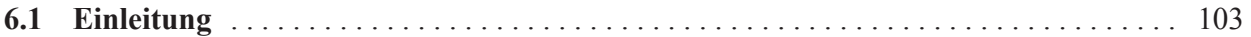

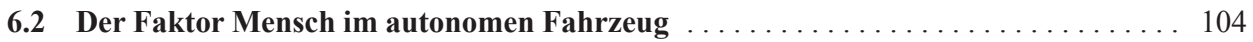

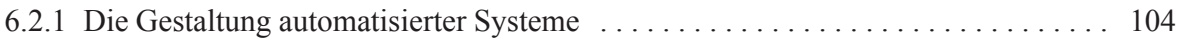

6.2 .2 Automation im Auto . . . . . . . . . . . . . . . . . . . . . . . . 106

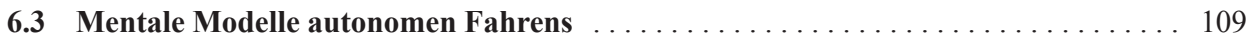

6.3.1 Was sind mentale Modelle? . . . . . . . . . . . . . . . . . . . . . . . . . . . . 109

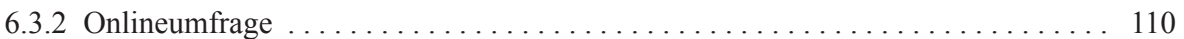

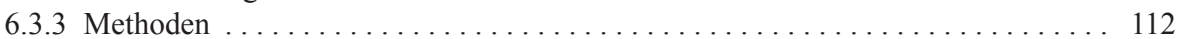

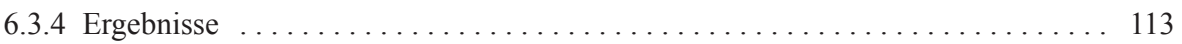

6.4 Zusammenfassung und Schlussfolgerung $\ldots \ldots \ldots \ldots \ldots \ldots \ldots \ldots \ldots \ldots \ldots \ldots$

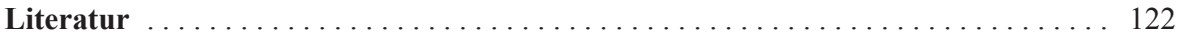

\subsection{Einleitung}

Menschen repräsentieren Wissen und Lernerfahrungen in Form von mentalen Modellen. Dieses aus der Kognitionspsychologie stammende Konzept ist eines der zentralen theoretischen Paradigmen für das Verständnis und die Gestaltung der Interaktion von Menschen mit technischen Systemen [1]. Mentale Modelle dienen in diesem Kontext einerseits der Beschreibung menschlicher Informationsverarbeitung, z. B. zur Beantwortung der Fragen, wie schnell eingehende Informationen wahrgenommen und gespeichert werden, oder

I. Wolf $(\bowtie)$

Freie Universität Berlin, Institut Futur, Deutschland wolf@institutfutur.de 
welche Informationen der Denkapparat eines Menschen benötigt, um auf veränderte Umweltbedingungen adäquat zu reagieren. Andererseits können auf diese Weise Wissensrepräsentationen und Funktionsannahmen konzeptualisiert werden, um beispielsweise Erwartungen und Verhaltensweisen von Nutzern im Umgang mit automatisierten Systemen zu verstehen und vorherzusagen.

Die Automatisierung der Fahrzeugführung verändert die Anforderungen an das kognitive System des Autofahrers grundlegend. Die Rolle des Menschen als physisch aktiver Entscheidungsträger im Fahrzeug wird mit zunehmendem Automatisierungsgrad durch automatisierte Systeme ersetzt. Bislang wichtige Handlungsmuster (z. B. zur Durchführung von Lenkmanövern) werden nicht mehr benötigt und unter Umständen verlernt, gleichzeitig müssen neue Fertigkeiten (z. B. Systemüberwachung) und ein neues Systemverständnis erlernt werden. Zugrundeliegende mentale Modelle müssen modifiziert oder neu strukturiert werden. Für die Sicherheit und die Akzeptanz von autonomen Fahrzeugen wird es von entscheidender Bedeutung sein, die neuen Rollen des Menschen im autonomen Fahrzeug so zu definieren, dass sie sowohl den Fähigkeiten des menschlichen Informationsverarbeitungssystems entsprechen, als auch den Erwartungen und Bedürfnissen der Menschen gerecht werden. In dem vorliegenden Beitrag werden diese beiden Aspekte betrachtet. Mit Blick auf die bisherigen Erkenntnisse der Automatisierung in unterschiedlichen Domänen wird der Frage nachgegangen, welche kognitiven und emotionalen Dimensionen bei der Gestaltung von automatisierten Fahrzeugen zu berücksichtigen sind. Auf Basis einer mit den Co-Autorinnen dieses Buches Rita Cyganski, Eva Fraedrich und Barbara Lenz durchgeführten deutschlandweiten Online-Befragung wird komplementär dazu untersucht, mit welchen mentalen Modellen potenzielle Nutzer autonomen Fahrzeugen begegnen.

Das Kapitel ist in zwei Hauptabschnitte unterteilt. Im ersten Teil wird zunächst ein Überblick zu den zentralen Modellen, Gestaltungskonzepten und Befunden der Automatisierung in Hinblick auf die Herausforderungen und Problembereiche der Mensch-Maschine-Interaktion gegeben. Dem folgt eine Zusammenfassung der bisherigen Ergebnisse zu den kognitiven Auswirkungen in (teil-)automatisierten Fahrzeugen. Im Anschluss wird der theoretische Hintergrund des Konzepts der mentalen Modelle erläutert. Der zweite Teil widmet sich den Ergebnissen der Online-Befragung. Die Mobilitäts-, Kontroll- und Erlebnisbedürfnisse sowie emotionalen Reaktionen potenzieller Nutzer gegenüber autonomen Fahrzeugen werden dabei, differenziert nach den in dem Projekt entwickelten Nutzungsszenarien, dargestellt. Das Kapitel schließt mit einer Zusammenfassung und den Schlussfolgerungen der Ergebnisse.

\subsection{Der Faktor Mensch im autonomen Fahrzeug}

\subsubsection{Die Gestaltung automatisierter Systeme}

Die Frage der nutzergerechten Gestaltung automatischer Systeme ist seit Jahrzehnten Gegenstand wissenschaftlicher Diskussion (vgl. z. B. [2, 3]). Mit der wachsenden Leistungsfähig- 
keit technischer Systeme gewinnt dieser Aspekt zunehmend an Bedeutung. Erfahrungen aus unterschiedlichen Domänen, wie z.B. der Luftfahrt, mit der (Teil-)Automatisierung von technischen Systemen verdeutlichen, dass die Sicherheit und Zuverlässigkeit derartiger Systeme nicht allein durch die Optimierung technischer Komponenten erreicht werden kann. Vielmehr wird die Verlässlichkeit automatisierter Systeme maßgeblich von der Qualität der Interaktion zwischen Mensch und Maschine bestimmt. Dies gilt im Besonderen für Situationen, in denen dem Menschen die Aufgabe zukommt, Fehler des technischen Systems zu korrigieren und bei Ausfällen oder Störungen die Systemkontrolle zu übernehmen.

Im Zuge der Automatisierung erfolgt eine Verlagerung von Funktionen auf technische Systeme, die die Rolle und erforderlichen Kompetenzen des Menschen wesentlich verändert. So übernehmen in modernen Flugzeugcockpits Computersysteme (z. B. FlightManagement-System oder Autopilot) Aufgaben, die früher von der Cockpitbesatzung ausgeführt wurden. Die Anforderungen an den Piloten verschieben sich somit von aktiven manuellen Steuerungsaufgaben hin zu Tätigkeiten der Programmierung und Überwachung der Flugzeugautomation. Diese auch als leitende Kontrolle (supervisory control, [4]) bezeichnete Überwachungsfunktion des Menschen führte z. B. in der Luftfahrt zur Entlastung der Piloten und bedeutsamen Steigerung der Flugsicherheit [5]. Gleichzeitig haben die psychologischen Folgen der passiven Rolle des Systemüberwachers wie reduzierte Aufmerksamkeit oder Aktivierung massive Sicherheitsprobleme verursacht [6]. Bainbridge [7] spricht in diesem Zusammenhang von der „Ironie der Automatisierung“ - einerseits werden Systemfunktionen aufgrund der Fehlerhaftigkeit des Menschen automatisiert, und anderseits soll genau dieser Mensch das System überwachen und im Notfall als Rückfalloption zur Verfügung stehen.

Die aus dem Gestaltungskonzept der leitendenden Kontrolle entstehenden Probleme sind in dem Forschungsgebiet „Human Factors“ umfänglich dokumentiert und werden unter dem Begriff „Out-of-the-loop-unfamiliarity“ zusammengefasst (OOTLUF, [8]). Die negativen Folgen der Entkoppelung des Menschen von der direkten Steuerung und Kontrolle beziehen sich insbesondere auf drei Aspekte, die in unterschiedlichen Anwendungskontexten identifiziert wurden: mangelndes bzw. übersteigertes Vertrauen in die Automation [9], Verlust manueller und kognitiver Fertigkeiten [10] sowie Schwierigkeiten bei der Aufrechterhaltung eines angemessenen Situations- und Systembewusstseins [11]. Unangemessenes Vertrauen kann darin resultieren, dass automatisierte Systeme nur unzureichend überwacht oder genutzt werden. Das Vertrauen in eine Automation wird von der Zuverlässigkeit, der Nachvollziehbarkeit und der wahrgenommenen Nützlichkeit beeinflusst. Die Folgen des Verlusts manueller und kognitiver Fertigkeiten werden hingegen erst dann salient, wenn der Nutzer im Falle eines Automationsfehlers plötzlich gezwungen ist, automatisierte Funktionen wieder selbst zu übernehmen. Mangelndes Training und Ausübung von Tätigkeiten kann zu Effektivitätseinbußen sowohl in motorischen als auch in kognitiven Bereichen führen. Die „Out-of-the-loop“-Effekte treten besonders hinsichtlich der Wahrnehmung und richtigen Interpretation der Systemprozesse - dem sogenannten Situationsbewusstsein - auf. Die Gründe für mangelndes Situationsbewusstsein liegen vornehmlich in der unzureichenden Überwachung des Systems, dem veränderten oder kompletten 
Wegfall von Rückmeldungen (z. B. taktile Reize vom Lenkrad), der mangelnden Transparenz der Automation und dem komplexitätsbedingten mangelnden Systemverständnis. Aus kognitionspsychologischer Sicht fehlen den Menschen die entsprechenden mentalen Modelle (d.h. Wissens- und Fertigkeitsstrukturen), um die Funktionsweise der Automation zu verstehen [12].

Infolge der negativen Erfahrungen aus den technikzentrierten Gestaltungsansätzen kam es zu einem Umdenken in der Systemgestaltung. Unter dem Primat, den Menschen in-the-loop zu halten, indem Kontrollierbarkeit, Transparenz und Vorhersagbarkeit gewährleistet werden, hat sich das Konzept der menschzentrierten Automatisierung als zentrales Gestaltungsprinzip automatisierter Systeme weitgehend durchgesetzt (vgl. z. B. [13,14]). Grundlegende Prämisse dabei ist, dass der Mensch unabhängig vom Grad der Automatisierung die letztendliche Verantwortung für das Gesamtsystem trägt. Mensch und Maschine werden in diesem Kontext metaphorisch als Kooperationspartner betrachtet [15]. Gestaltungskonzepte der adaptiven Automation denken diesen Aspekt noch weiter und weisen Funktionen zwischen Mensch und Maschine dynamisch in Abhängigkeit situativer Erfordernisse zu [16]. Umfangreiche Forschungen zu der Anwendung dieser Designstrategien haben die Vorteile, aber auch die damit verbundenen Schwierigkeiten und zukünftigen Herausforderungen verdeutlicht (vgl. z. B. [17]).

Die steigende Komplexität und Autonomie sozio-technischer Systeme stellt jedoch die Sinnhaftigkeit des Primats menschlicher Verantwortung und Kontrolle infrage und wirft gleichzeitig für bestehende Konzepte das Problem auf, die Interaktion zweier autonom entscheidender Systemelemente - Mensch und Maschine - konfliktfrei zu gestalten [18,19]. Der menschzentrierte Gestaltungsansatz bedarf folglich einer weiterreichenden Weiterentwicklung oder Erneuerung [20], die gegebenenfalls erst durch eine breite gesellschaftliche Diskussion zu grundlegenden Fragen hinsichtlich der erwünschten Rolle der Automation im Lebensalltag beantwortet werden kann [21]. Nutzungskontexte und -häufigkeiten sowie die Kompetenzen und Expertise der Nutzer variieren jedoch über die unterschiedlichen Domänen erheblich, sodass für den Fahrzeugbereich spezifische Gestaltungskonzepte benötigt werden, die der Heterogenität der Autofahrer gerecht werden.

\subsubsection{Automation im Auto}

Auch im Fahrzeugbereich schreitet der Rollenwandel des Menschen vom aktiven Operateur hin zum passiven Überwacher des Systems immer weiter voran. Die mediale Berichterstattung zum Thema autonomes Fahren vermittelt den Eindruck, dass bereits in naher Zukunft fahrerlose Fahrzeuge die Sicherheit auf unseren Straßen verbessern werden (vgl. z.B. [22]). Obgleich bereits heute einzelne Funktionen in Fahrzeugen durch automatisierte Systeme wie z. B. den Abstandsregeltempomat übernommen werden, wird die Technologie jedoch in absehbarer Zeit nicht ohne die Verfügbarkeit des Menschen auskommen, der weiterhin Kontrollfunktionen und strategische Entscheidungen übernehmen wird [23]. 
Bislang ungeklärt ist die Frage, wie die Rolle des Menschen auf dem Weg hin zum vollständig autonomen Fahrzeug psychologisch sinnvoll und nutzergerecht definiert werden kann. Die oben dargestellten Erkenntnisse und Erfahrungen aus dem Bereich der Luftfahrt stellen zwar einen wichtigen Ausgangspunkt für die Beantwortung dieser Frage dar, sind jedoch aufgrund der höheren Umgebungskomplexität und -dynamik im Straßenverkehr nur bedingt für Gestaltungskonzepte im Fahrzeug nutzbar. Eine zunehmende Anzahl an Forschungsarbeiten hat sich in den letzten Jahren mit der Wechselwirkung zwischen teil- und hochautomatisierten Fahrfunktionen und dem menschlichem Verhalten beschäftigt (vgl. [24, 25]). Im Zentrum der Betrachtung stehen auch hier über unterschiedliche Automatisierungsstufen hinweg die bekannten Problembereiche der Automatisierung: Vertrauen, Kompetenzverlust und Situationsbewusstsein.

Automation ist nur dann sinnvoll, wenn Operateure dem technischen System vertrauen und es folglich auch nutzen. Die zentrale Herausforderung in der Gestaltung automatisierter Systeme ist es, angemessenes Vertrauen in diese Systeme zu erzeugen. Dabei können Fehler der Automation zum Rückgang des Vertrauens [26] führen. Dagegen kann übersteigertes Vertrauen zur Folge haben, dass die Automation nur unzureichend überwacht und kontrolliert wird (overtrust oder complacency, [27]). Der Großteil der bisherigen Forschungsarbeiten in diesem Kontext beschäftigt sich mit den Wechselwirkungen von Vertrauen im Umgang mit Adaptive Cruise Control (ACC; auf Deutsch: adaptive Geschwindigkeitsregelung). Ein gewisses Maß an Vertrauen kann dabei bereits eine wichtige Voraussetzung für die Nutzungsbereitschaft von Fahrerassistenzsystemen sein [28]. Kazi et al. [29] untersuchten in einer Längsschnittstudie im Fahrsimulator den Einfluss der Zuverlässigkeit von ACC auf das wahrgenommene Vertrauen in diese Systeme. Die Ergebnisse zeigen eine Vertrauenszunahme bei zuverlässigen Systemen im Verlauf der Zeit, die jedoch nicht im Verhältnis zur objektiven Zuverlässigkeit der Automation stand. Zu ähnlichen Ergebnissen kommen Koustanaï et al. [30], die durch die systematische Graduierung des Erfahrungsstandes im Umgang mit Auffahrwarnsystemen Veränderungen im Verhalten und im Vertrauen untersuchten. Die Teilnehmergruppe mit dem höchsten Erfahrungsstand hatte im Simulator keine Unfälle und reagierte in kritischen Situationen angemessener als die Fahrer mit geringerem Erfahrungsniveau. Ebenso stand der Grad der Systemerfahrung im positiven Zusammenhang mit dem geäußerten Vertrauen, jedoch ohne die Akzeptanz der Automation zu beeinflussen. Im Kontrast zu diesen Befunden stehen die Ergebnisse mehrerer Studien, die keine signifikanten Veränderungen des Vertrauens in ACC durch wiederholte Nutzung feststellen konnten (vgl. z. B. [31, 32]). Ursachen dieser inkonsistenten Ergebnisse könnten moderierende Faktoren sein, die in jüngsten Arbeiten betrachtet wurden. Flemisch et al. [33] und Beggiatio et al. [34] heben in diesem Kontext die Bedeutung übereinstimmender (bereits vorab ausgebildeter) mentaler Modelle über die Funktionsweise der jeweiligen Automation hervor. Noch einen Schritt weiter gehen Verberne et al. [35] und Waytz et al. [36]. Auf der Basis experimenteller Studien zeigen sie, dass geteilte Intentionen und Bedürfnisse zwischen Mensch und Maschine bzw. anthropomorphe Eigenschaften der Automation weitere wichtige Einflussfaktoren für die Ausbildung angemessenen Vertrauens in automatisierte Systeme darstellen können. 
Das Führen eines Fahrzeugs verlangt vom Fahrer eine große Spannbreite an Kompetenzen und Fertigkeiten sowohl auf perzeptuell-motorischer Ebene (z. B. Lenken, Schalten etc.), als auch auf kognitiver Ebene (z. B. Entscheidungen treffen, Aufmerksamkeit selektiv richten etc.). Die automatisierte Ausführung dieser Aufgaben kann zum Verlust dieser Kompetenzen führen und gleichzeitig die Abhängigkeit vom technischen System erhöhen [37]. Die grundsätzliche Brisanz des Themas wird anhand einer aktuellen Sicherheitsmitteilung der Bundesluftfahrbehörde der Vereinigten Staaten (Federal Aviation Administration, [38]) deutlich. Darin werden Piloten aufgefordert, häufiger den manuellen Flugmodus anstatt den Autopiloten zu wählen, da der Kompetenzverlust ein zunehmendes Sicherheitsrisiko für die Luftfahrt darstellt. Obgleich nach Kenntnis des Autors aktuell keine longitudinale Fehlstudien zu den Problemen des Fertigkeitsverlusts in (teil-)automatisierten Fahrzeugen vorliegen, ,,deuten die Ergebnisse erster Untersuchungen im Fahrsimulator darauf hin (z.B. [68], “ dass diese Effekte auch im Kontext der Fahrzeugautomatisierung auftreten. Adaptive oder kooperative Automatisierungskonzepte bieten die Möglichkeit, derartigen Problemen entgegenzuwirken und bis zur Realisierung vollständig autonomer Fahrzeuge wichtige Kompetenzen der Fahrer weiterhin aufrechtzuerhalten.

Der Fähigkeit, komplexe und dynamische Fahrsituationen richtig wahrzunehmen und zu interpretieren, liegt eine Reihe von kognitiven Prozessen (z. B. Aufmerksamkeit, Gedächtnis, mentale Modelle) zugrunde [12]. Monotone Überwachungsaufgaben oder die Ablenkung durch andere Tätigkeiten (z. B. Telefonieren) können dazu führen, dass diese Prozesse nicht mehr in ausreichendem Maße für das Situationsbewusstsein im Fahrzeug zur Verfügung stehen. Diese Effekte können bereits durch die Nutzung von Systemen mit niedrigem Automatisierungsgrad wie Adaptive Cruise Control (ACC) auftreten. Buld et al. [39] konnten zeigen, dass Fahrer während der Nutzung von ACC einige Aspekte der Fahraufgabe und Umgebungsbedingungen vernachlässigten und in Folge die Systemgrenzen falsch interpretierten. Verschlechterte Spurhaltung und verspätete Reaktion auf kritische Ereignisse wurden in einer Untersuchung von Ward [40] als Indikatoren reduzierten Situationsbewusstsein beim Fahren mit ACC interpretiert. Die Analysen von Ma und Kaper [41] deuten jedoch darauf hin, dass sich das Situationsbewusstsein infolge der Nutzung von ACC auch verbessern kann. Ein differenzierteres Bild dieser widersprüchlichen Ergebnisse liefern aktuelle Studien zu den Folgen hochautomatisierten Fahrens. Merat et al. [42] haben in einer Simulationsstudie die Effekte der Ausführung einer Nebenaufgabe auf das Fahrverhalten automatisierter Fahrt untersucht. Dabei zeigte sich, dass die Reaktionen auf kritische Ereignisse unter der hochautomatisierten und der manuellen Fahrbedingung ohne Nebentätigkeit vergleichbar waren. Während hingegen die Ablenkung durch Nebentätigkeiten dazu führte, dass nach der manuellen Übernahme der automatisierten Fahrt deutlich höhere Geschwindigkeiten gefahren wurden. Die Autoren führen die Ergebnisse auf das reduzierte Situationsbewusstsein zurück, das durch die nebentätigkeitsbedingte Aufmerksamkeitsverschiebung verursacht wird.

Die hier dargestellten Problembereiche stellen nur einen Ausschnitt der zu lösenden Herausforderungen im Zusammenspiel zwischen Mensch und automatisiertem Fahrzeug dar. Viele Fragen hinsichtlich der mentalen Anpassungen und Veränderungen werden erst durch konkrete technische Umsetzung und wissenschaftliche Untersuchung der nächst 
höheren Stufen der Fahrzeugautomatisierung (vgl. Automatisierungsstufen BASt, [43]) zu beantworten sein. Die Gestaltung von Schnittstellen, geeignetes Feedback oder die Vermeidung von Verantwortungsdiffusion sind Themenfelder, die bereits heute in neuen Gestaltungskonzepten adressiert werden und auf Prototypenbasis für hochautomatisierte Fahrzeuge umgesetzt wurden (vgl. z. B. [44]). Welche Lernerfahrungen, Wechselwirkungen und Veränderungen mentaler Modelle sich jedoch langfristig durch die Nutzung dieser Systeme ergeben, kann erst durch repräsentative, longitudinale Untersuchungen geklärt werden.

\subsection{Mentale Modelle autonomen Fahrens}

\subsubsection{Was sind mentale Modelle?}

Als mentale Modelle werden kognitiv-emotionale Repräsentationen von Objekten, Objektbeziehungen und Prozessen - kurz: innere Repräsentationen der externen Welt - bezeichnet. Der Begriff des mentalen Modells wurde erstmals von dem Psychologen Kenneth Craik [45] verwendet, der die Annahme formulierte, dass Menschen in ihrem Geist vereinfachte Modelle über die Funktionen und Abläufe ihrer Umwelt entwickeln. Die Modelle dienen der Orientierung, dem Verständnis, dem Schlussfolgern sowie der Vorhersage von Ereignissen. Craiks Ansatz der mentalen Modelle wurde später von Johnson-Laird [46] zur Beschreibung und Untersuchung von schlussfolgerndem Denken und Sprachverstehen weiterentwickelt.

In der Literatur der Kognitionspsychologie besteht weitgehend Konsens darüber (vgl. [47]), dass mentale Modelle dynamischer Natur sind und sich durch drei zentrale Eigenschaften beschreiben lassen.

1. Mentale Modelle werden im Arbeitsgedächtnis erstellt und ermöglichen Individuen, Handlungsmöglichkeiten und ihre Folgen zu simulieren [1]. Denken ist somit die Manipulation von mentalen Modellen.

2. Mentale Modelle können Ursache und Wirkungszusammenhänge repräsentieren.

Sie generieren ein kausales Verständnis darüber, wie Systeme funktionieren [48].

3. Mentale Modelle können sich durch Erfahrungen im Verlauf der Zeit verändern d.h., sie sind lernfähig.

Die Qualität der Modelle und die der darauf basierenden Schlussfolgerungen entwickeln sich durch spezifische Lernerfahrungen weiter [49]. Mit zunehmender Expertise entwickelt sich das Verständnis von Sachverhalten von konkreten hin zu abstrakten Repräsentationen - ein für die Mensch-Maschine-Interaktion relevanter Aspekt.

In den angewandten Forschungsbereichen wie der Technikgestaltung herrschen teilweise unterschiedliche Auffassungen über die Definition mentaler Modelle (vgl. [1]), die durch unterschiedliche Aufgabenkontexte erklärbar sind. Dennoch haben bereits frühere Arbeiten die Bedeutung des Konzeptes zur Vorhersage und zum Verständnis menschlichen Verhaltens im Umgang mit technischen Systemen verdeutlicht (vgl. z. B. [50]). Mentale 
Modelle basieren demnach auf kontextspezifischen Erwartungen und Vorerfahrungen sowie auf der aktuellen Wahrnehmung der Systemeigenschaften. Sie stellen die Grundlage des Systemverständnisses und der Handlungsentscheidungen des Benutzers dar. Dies bedeutet, dass sowohl die fehlerfreie Nutzung als auch das Vertrauen in technische Systeme maßgeblich davon bestimmt wird, inwieweit die Funktionsweise der Maschine mit den Erwartungen der Nutzer kompatibel ist [33].

Die Kompatibilität im Kontext mentaler Modelle bezieht sich neben der Bedienbarkeit auch auf das Nutzererleben sowie die allgemeine Technikakzeptanz. Zhang und Xu [51] postulieren in diesem Zusammenhang eine Modifizierung oder Restrukturierung bestehender mentaler Modelle bei der Einführung und Nutzung neuer Technologien. Mangelnde Kompatibilität kann zur Frustration führen und beeinflusst folglich Akzeptanz- und Diffusionsraten negativ [52]. Entsprechen hingegen neue Systeme den Erwartungen (d.h. den bestehenden mentalen Modellen), hat dies gesteigertes Systemvertrauen und positives Nutzererleben zur Folge [53].

Mentale Modelle umfassen folglich Repräsentationen menschlichen Wissens, Einstellungen sowie Werte und Emotionen, die in Wechselwirkung mit ihrer Umwelt stehen. Im Themenfeld der Automatisierung von Fahrzeugen sind sowohl die kognitionspsychologischen Prozesse der Informationsverarbeitung als auch der Einfluss höherer mentaler Strukturen (z. B. Bedürfnisse, Erwartungen, Wünsche etc.) von Bedeutung. Die Interdependenz dieser unterschiedlichen Ebenen wurde bereits in theoretischen Modellen über die Rolle des Fahrers in automatisierten Fahrzeugen verdeutlicht (vgl. z. B. [54, 55]). Letztendlich wird die geeignete Modifikation und Anpassung der mentalen Modelle den Umgang mit automatisierten Fahrzeugen, die Nutzungshäufigkeit sowie die Akzeptanz dieser Systeme maßgeblich bestimmen. Der erfolgreiche - noch zu definierende - Rollenwandel des Fahrers im automatisierten Fahrzeug bedarf somit einer integrativen Betrachtung der vorliegenden Ergebnisse zu menschlichem Verhalten in teil- und hochautomatisierten Systemen sowie der aktuellen Vorstellungen und Bedürfnisse gegenüber vollautomatisierten Fahrzeugen. Oder anders gesagt, menschzentrierte Technikgestaltung impliziert nicht nur die Betrachtung der technischen Möglichkeiten und Grenzen, sondern auch die Orientierung an individuellen und gesellschaftlichen Wertevorstellungen und Zielen.

\subsubsection{Onlineumfrage}

Für viele Menschen sind autonome Fahrzeuge noch eine ferne Zukunftsvision. Auch wenn sich manche bereits vorgestellt haben mögen, wie attraktiv es wäre, während der Autofahrt zu schlafen oder Zeitung zu lesen, ist das Wissen über autonome Fahrzeuge in der breiten Bevölkerung noch begrenzt. Entscheidungen über die Nutzung und Akzeptanz von Innovationen basieren jedoch nicht ausschließlich auf rationalem Wissen [56]. Entgegen dem Menschenbild des rationalen, nutzenmaximierenden Entscheiders - dem homo oeconomicus - bedienen sich Menschen vielmehr einfacher Entscheidungsstrategien, welche die zu verarbeitende Informationsmenge reduzieren und von emotionalen Prozessen beein- 
flusst werden $[57,58,59]$. Einstellungen und Entscheidungen sind nicht beliebig durch die Bereitstellungen von Informationen veränderbar. Vielmehr werden neue Informationen selektiv aufgenommen und verarbeitet, sodass sie im Einklang mit den bestehenden Wünschen, Erwartungen und Zielen - den mentalen Modellen der Menschen - stehen [60]. Für den Erfolg einer Innovation ist es folglich entscheidend, dass nicht nur deren kognitive Wahrnehmungen und Bewertungen in bestehende mentale Modelle integrierbar, sondern auch emotional anschlussfähig sind [61, 62].

Neben einer Vielzahl von Studien zu den technischen, rechtlichen und kognitiven Aspekten der Automatisierung von Fahrzeugen existieren bislang nur wenige Untersuchungen, die sich mit den Präferenzen und Erwartungen potenzieller Nutzer auseinandersetzen. In der bislang größten repräsentativen internationalen Erhebung zu dem Thema [63] standen insbesondere die Akzeptanz und Nutzungsbereitschaft automatisierter Fahrzeuge im Fokus des Interesses. Die Ergebnisse zeigen für Deutschland, dass automatisierte Fahrzeuge mehrheitlich als nützlicher technischer Fortschritt erachtet werden. Dagegen äußert gleichzeitig die Hälfte der Teilnehmer Angst gegenüber dem automatisierten Fahren und bezweifelt, dass die Technologie zuverlässig funktionieren wird. Im Vergleich mehrerer Nutzungsszenarien werden insbesondere lange Autobahnfahrten als bevorzugte Einsatzmöglichkeit des autonomen Fahrens genannt. Interessanterweise finden die Autoren einen positiven Zusammenhang zwischen der Akzeptanz von Fahrerassistenzsystemen und der Akzeptanz gegenüber dem automatisierten Fahren. Eine mögliche Erklärung dafür könnte sein, dass die Ausbildung geeigneter mentaler Modelle zu den Eigenschaften teilautomatisierter Systeme auch das Akzeptanzniveau für höhere Automatisierungsstufen positiv beeinflussen (vgl. [34]).

Welche Einstellungen sowie kognitiven und emotionalen Repräsentationen der Akzeptanz oder Ablehnung automatisierter Fahrzeuge zugrunde liegen, ist bislang unbekannt. In Ergänzung zu den oben dargelegten kognitionspsychologischen Anforderungen an die Gestaltung der Mensch-Maschine-Interaktion stellen diese jedoch eine wichtige Voraussetzung für das Gelingen der Transformation im Verkehrssektor dar. Ziel der hier dargestellten repräsentativen Onlinefragebogenstudie war es, ein differenziertes, teilweise exploratives Bild der Wahrnehmungen des autonomen Fahrens über die in dem Projekt entwickelten Use-Cases hinweg zu generieren. Der Fragebogen wurde unter den folgenden Leitfragen entwickelt:

- „Mit welchen mentalen Modellen begegnen potenzielle Nutzer der neuen Rolle des Fahrers im autonomen Fahrzeug?"

- „Welche automatisierten Elemente der Fahrzeugsteuerung sind am ehesten an die mentalen Modelle der Nutzer anschlussfähig?“

- „Welche Kontrollfunktionen und Interventionsmöglichkeiten des Fahrers erwarten potenzielle Nutzer im autonomen Fahrzeug bzw. können die Akzeptanz des Innovationsfeldes steigern?"

- „Welche Erlebnis- und Gestaltungselemente in automatisierten Fahrzeugen können bisherige Repräsentationen zur Rolle des Fahrers substituieren und somit die Akzeptanz des Innovationsfeldes steigern?““ 


\subsubsection{Methoden}

\subsubsection{Fragebogen}

Der Fragebogen wurde in Zusammenarbeit mit weiteren Autorinnen dieses Buches (Rita Cyganski, s. Kap. 12 sowie Eva Fraedrich und Barbara Lenz, s. Kap. 29) konzipiert. Die Erhebung erfolgte internetbasiert im April 2014 über einen elektronischen Fragebogen. Der Fragebogen war in zwei Hauptabschnitte unterteilt:

1. Allgemeiner Teil: Dieser Bereich bestand aus fünf Fragengruppen, die Fragen zur Soziodemografie, zu den Vorkenntnissen, dem Interesse und der allgemeinen Akzeptanz automatisierten Fahrens, zu bedürfnisbezogenen Einstellungen bezüglich unterschiedlicher Verkehrsmittel, zu den emotionalen Repräsentationen mobilitätsbezogener Begriffe sowie den Themen der Zeitnutzung und zur allgemeinen Verkehrsmittelnutzung umfassen.

2. Spezieller Teil: Die Fragen in diesem Teil bezogen sich auf die im Projekt entwickelten vier Nutzungsszenarien (sogenannte Use-Cases, s. Kap. 2) und waren jeweils in folgende zehn Themengruppen unterteilt: freie Assoziationen zu dem Nutzungsszenario, Nutzungsbereitschaft, antizipierter Wegzweck, antizipierter Einfluss auf bisherige Verkehrsmittelnutzung, angenommene Bedürfniserfüllung, emotionale Reaktionen, Vertrauen und Akzeptanz, Kontroll- und Interventionsbedürfnisse sowie bevorzugte Nebenaufgaben während der automatisierten Fahrt.

Zur Reduzierung der Bearbeitungszeit wurden die Fragen zu den vier unterschiedlichen Use-Cases (s. unten 6.3.2.2-6.3.2.4) im zweiten Teil nicht von jedem Teilnehmer beantwortet. Nach der Beantwortung der Fragen im ersten Teil wurde das Sample gesplittet und die Studienteilnehmer zufällig und zu gleichen Teilen (jeweils $n=250$ ) einem der vier Nutzungsszenarien zugewiesen. Insgesamt umfasste der Fragebogen 438 Items, wobei durch die Aufteilung der Use-Cases von jedem Teilnehmer 210 Fragen beantwortet wurden. Die Items wurden zum Teil aus früheren Mobilitätserhebungen [62, 64] übernommen bzw. neu entwickelt und - insbesondere die Fragen des zweiten Teils - in einem Pretest auf Verständlichkeit geprüft.

Bei allen Einstellungsfragen wurde der Grad der Zustimmung über eine sechsstufige Skala ( 1 = trifft überhaupt nicht zu, $6=$ trifft voll und ganz zu; bei einigen Fragen weichen diese Codes aus inhaltlichen Gründen ab) erfasst. Die affektive Bedeutung der Begriffe im Themenfeld Mobilität wurde mit der Methode des semantischen Differenzials erhoben [65]. In den drei Dimensionen Valenz, Potenz und Erregung wurden dazu jeweils bipolare, neunstufige (von $-4=\ddot{a} u ß e r s t$ über $0=$ neutral bis zu $4=\ddot{a} u ß e r s t)$ Skalen verwendet, die an den Endpunkten mit den Adjektiven unangenehm - angenehm (Valenz), schwach mächtig (Potenz) und beruhigend - aufregend (Erregung) gekennzeichnet waren. Das aktuelle Verkehrsverhalten wurde über Auswahloptionen bzw. Häufigkeitskategorien erfasst. 


\subsubsection{Stichprobe}

Die Teilnehmer der Befragung wurden über ein kommerzielles Marktforschungspanel der Firma Respondi AG rekrutiert (http://www.respondi.com/de/) und von dieser für ihr Mitwirken finanziell entlohnt. Von dem Unternehmen wurde eine für die gesamtdeutsche Bevölkerung repräsentative Zusammensetzung hinsichtlich Alter, Geschlecht, Bildung und Einkommen zugesichert. Insgesamt haben $n=1363$ Personen den Fragebogen vollständig ausgefüllt. Einige Personen beantworteten die Fragen jedoch in so kurzer Zeit, dass ihre Gewissenhaftigkeit in Zweifel gezogen werden muss. Folglich wurden die Daten all jener Teilnehmer, deren Bearbeitungszeit unter 1000 Sekunden lag, in den weiteren Analysen nicht weiter berücksichtigt. Das Sample reduzierte sich somit um $n=230$ auf $n=1133$. In einem weiteren Schritt wurden infolge der sich daraus ergebenden Quotenverzerrung $n=133$ weibliche Personen zufällig aus den Datensatz entfernt, um eine annähernd repräsentative Verteilung zumindest in der Geschlechterquote zu erreichen. Die mittlere Bearbeitungszeit der verbleibenden Stichprobe ( $\mathrm{n}=1000)$ lag bei 1897 Sekunden (=31,6 Minuten) $(\mathrm{SD}=780$ Sekunden). Tabelle 6.1 ist die genaue demografische Zusammensetzung des Samples zu entnehmen.

\subsubsection{Datenanalyse affektive Ähnlichkeit}

Die affektive Ähnlichkeit zwischen den mittels semantischen Differenzials bewerteten Begrifflichkeiten wurde durch die dreidimensionale euklidische Distanz zwischen dem gemittelten EPA-Profil ( $\mathrm{E}=$ Valenz, $\mathrm{P}$ = Potenz, A = Erregung) des Begriffes „Ideale Fahrt“ und den gemittelten EPA-Profilen der übrigen Begriffe in folgender Weise berechnet:

$$
d=\sqrt{\left(I_{e}-B_{e}\right)^{2}+\left(I_{p}-B_{p}\right)^{2}+\left(I_{a}-B_{a}\right)^{2}},
$$

wobei $I$ die Bewertung der „Idealen Fahrt“ bezeichnet, $B$ die jeweilige Bewertung der übrigen Begriffe und die tiefgestellten Buchstaben die EPA-Dimensionen festlegen.

\subsubsection{Ergebnisse}

Zunächst interessierte, inwieweit das Thema „Autonomes Fahren“ in der Bevölkerung überhaupt bekannt ist, ob es auf breites Interesse stößt und wie die Technologie spontan beurteilt wird. Weniger als die Hälfte der Befragten (44 Prozent) gab an, keine Kenntnisse von dem Thema zu haben, während die Mehrheit bereits von dem Thema gehört (33 Prozent), darüber gelesen hat (16 Prozent) oder nach eigenen Angaben über ein höheres Kompetenzniveau (4 Prozent) verfügt. Eine ähnliche Verteilung zeigt sich auch hinsichtlich des Interesses am Thema „Autonomes Fahren“. Die Teilnehmer äußerten mehrheitlich (58 Prozent) „leichtes“, „überwiegendes“ oder ,starkes“ Interesse an dem Thema, während jedoch die Mehrheit (56 Prozent) sich grundsätzlich nicht vorstellen kann, das eigene bislang bevorzugte Verkehrsmittel durch ein autonomes Fahrzeug zu ersetzen. Trotz überwiegenden Interesses und einiger Vorkenntnisse besteht also bei einem Großteil der Be- 
Tab. 6.1 Demografische und mobilitätsspezifische Merkmale der Stichprobe

\begin{tabular}{|c|c|c|}
\hline \multicolumn{3}{|l|}{ Merkmale } \\
\hline \multicolumn{3}{|l|}{ Demografie } \\
\hline Geschlecht & weiblich & $55,5 \%$ \\
\hline \multirow{4}{*}{ Alter } & 18-29 Jahre & $8,8 \%$ \\
\hline & 30-49 Jahre & $33,6 \%$ \\
\hline & 50-64 Jahre & $31,7 \%$ \\
\hline & $65+$ Jahre & $25,9 \%$ \\
\hline \multirow[t]{4}{*}{ Bildung } & keine Schulausbildung & $1,1 \%$ \\
\hline & Volks-/Hauptschulabschluss & $39,4 \%$ \\
\hline & Mittlere Reife/Realschulabschluss & $29,5 \%$ \\
\hline & Abitur oder Fachhochschulreife & $30,0 \%$ \\
\hline \multirow[t]{6}{*}{ Einkommen } & unter 900 Euro pro Monat & $6,6 \%$ \\
\hline & 900 bis unter 1500 Euro pro Monat & $17,5 \%$ \\
\hline & 1500 bis unter 2000 Euro pro Monat & $15,2 \%$ \\
\hline & 2000 bis unter 2600 Euro pro Monat & $14,4 \%$ \\
\hline & 2600 bis unter 3600 Euro pro Monat & $18,6 \%$ \\
\hline & mehr als 3600 Euro pro Monat & $27,7 \%$ \\
\hline \multicolumn{3}{|l|}{ Mobilität } \\
\hline Führerschein & ja & $89,8 \%$ \\
\hline \multirow{4}{*}{$\begin{array}{l}\text { PKW-Anzahl } \\
\text { im Haushalt }\end{array}$} & kein Auto & $12,6 \%$ \\
\hline & 1 Auto & $51,8 \%$ \\
\hline & 2 Autos & $28,8 \%$ \\
\hline & 3 und mehr Autos & $6,8 \%$ \\
\hline \multirow{4}{*}{$\begin{array}{l}\text { Täglich genutztes } \\
\text { Verkehrsmittel }\end{array}$} & Auto & $55,0 \%$ \\
\hline & öffentlicher Nahverkehr & $13,7 \%$ \\
\hline & Carsharing & $0,4 \%$ \\
\hline & Fahrrad & $10,7 \%$ \\
\hline
\end{tabular}

völkerung eine gewisse Zurückhaltung hinsichtlich der Nutzungsbereitschaft autonomer Fahrzeuge.

\subsubsection{Fahrerassistenzsysteme und Abgabe von Fahraufgaben}

Wie bereits oben dargestellt, kann die Nutzung und Akzeptanz von Fahrerassistenzsystemen einen positiven Effekt auf die generelle Wahrnehmung des autonomen Fahrens haben. Die Ergebnisse der vorliegenden Studie zeigen, dass ein Großteil der Befragten (67 Prozent) bereits von Fahrerassistenzsystemen gehört hat. Unter den Personen, die im 


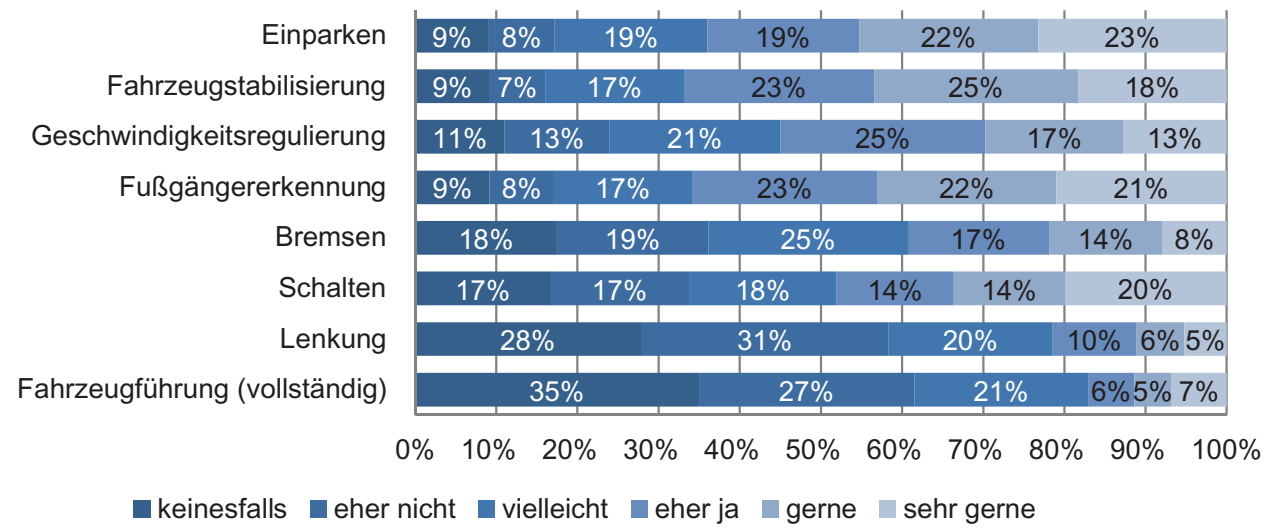

Abb. 6.1 Bedürfnis der Funktionsabgabe an ein automatisiertes System

Alltag einen Pkw nutzen (82 Prozent), wird am häufigsten der Tempomat (50 Prozent), die akustische Einparkhilfe (46 Prozent) und der Fernlichtassistent (22 Prozent) eingesetzt. Die übrigen Systeme wie Abstandsregeltempomat (ACC, 15 Prozent), Nachtsichtassistent (11 Prozent), Head-up-Display (10 Prozent) oder Müdigkeitsassistent (8 Prozent) werden nur von einer Minderheit im Alltag genutzt.

Die Bedürfnisse, bestimmte Fahraufgaben und -funktionen an ein automatisiertes System abzugeben, weisen ein ähnliches Bild auf. Abbildung 6.1 zeigt die aufgabenspezifische Verteilung der Bedürfnisse in dem Kategorienspektrum von „keinesfalls“ bis ,sehr gerne“. Im Vergleich der unterschiedlichen Fahraufgaben wird deutlich, dass neben der überwiegenden Ablehnung (62 Prozent in den Kategorien „keinesfalls“ und ,eher nicht“), die vollständige Fahrzeugführung an einen Fahrroboter abzugeben, insbesondere die Lenkung des Fahrzeugs (58,3 Prozent in den Kategorien ,keinesfalls“ und ,eher nicht“), ungern an ein automatisiertes System delegiert wird. Während die Übertragung von Einparkaufgaben (45 Prozent in den Kategorien ,gerne“ und „sehr gerne“) sowie sicherheitsrelevanter Assistenzen im Bereich der Fahrzeugstabilisierung (43 Prozent in den Kategorien ,gerne“ und „sehr gerne“) und der Fußgängererkennung (43 Prozent in den Kategorien „gerne“ und „sehr gerne") präferiert werden.

\subsubsection{Repräsentationen der Fahrerrolle und Nutzungsszenarien}

Auf der Basis des semantischen Differenzials wurden unter allen Teilnehmern die affektiven Bedeutungen unterschiedlicher Begriffe, die sich einerseits auf unterschiedliche Rollen im Fahrzeug und anderseits auf die in den Use-Cases beschriebenen Nutzungsszenarien beziehen, erhoben. Außerdem wurde der Begriff der „Idealen Fahrt“ und das konventionelle „Auto“ in dieser Art bewertet. Die Rohergebnisse (durchschnittliche Bewertungen auf den Skalen Valenz, Potenz und Aktivierung) sind in Tab. 6.2 dargestellt.

Die Ergebnisse wurden genutzt, um die euklidischen Distanzen und somit deren affektive Ähnlichkeit zwischen dem Begriff der „Idealen Fahrt“ und den übrigen Begriffen zu 
Tab. 6.2 Arithmetisches Mittel $(M)$ der affektiven Bewertungen

\begin{tabular}{|l|l|l|l|}
\hline Begriff & Valenz & Potenz & Erregung \\
\hline Chauffeur & 1,26 & 0,80 & $-0,05$ \\
\hline Beifahrer & 0,89 & $-0,06$ & 0,07 \\
\hline Passagier & 0,95 & 0,15 & 0,10 \\
\hline Co-Pilot & 0,61 & 0,34 & 0,37 \\
\hline Fahrzeug mit Autobahnpilot & 0,54 & 0,68 & 0,86 \\
\hline Fahrzeug mit Valet-Parken & 0,93 & 0,89 & 0,68 \\
\hline Vollautomatisiertes Fahrzeug & 0,26 & 0,68 & 1,00 \\
\hline Vehicle-on-Demand & $-0,69$ & $-0,05$ & 1,04 \\
\hline Auto & 2,23 & 1,65 & 0,85 \\
\hline Ideale Fahrt & 2,69 & 1,30 & $-0,42$
\end{tabular}

berechnen (für methodische Details (s. [61, 66]). Eine Visualisierung dieser Berechnungen bietet Abb. 6.2, in der auf der x-Achse die euklidische Distanz der bewerteten Begriffe abgebildet ist. Niedrige Werte indizieren eine geringere Distanz und somit höhere affektive Ähnlichkeit zwischen den Begriffen, d.h., sie lösen eine stärkere positive Assoziation bei den Befragten aus. Deutlich erkennbar ist, dass der „Chauffeur“ der „Idealen Fahrt“ affektiv am nächsten kommt, wobei der „Co-Pilot“ am wenigsten dieser emotionalen Repräsentation entspricht. Im Vergleich der unterschiedlichen Use-Cases des autonomen Fahrens wird sichtbar, dass das Vehicle-on-Demand deutlich von den Empfindungen für eine ideale Fahrt abweicht, wohingegen Fahrzeuge mit Valet-Parken am ehesten damit assoziiert sind. Die im Vergleich zu den Use-Cases deutlich positivere affektive Verankerung konventioneller Autos kann folglich ein wesentliches Akzeptanzhemmnis für die Einführung insbesondere vollautomatisierter Fahrzeuge darstellen. In Bezug auf die Rolle des Fahrers unterstreichen die gefundenen affektiven Repräsentationen die in einer weiteren Frage explizit adressierte Rollenpräferenz. Die Teilnehmer gaben bei diesem Item mittels Schie-

Abb.6.2 Euklidische Distanzen zur affektiven Repräsentation der „Idealen Fahrt“

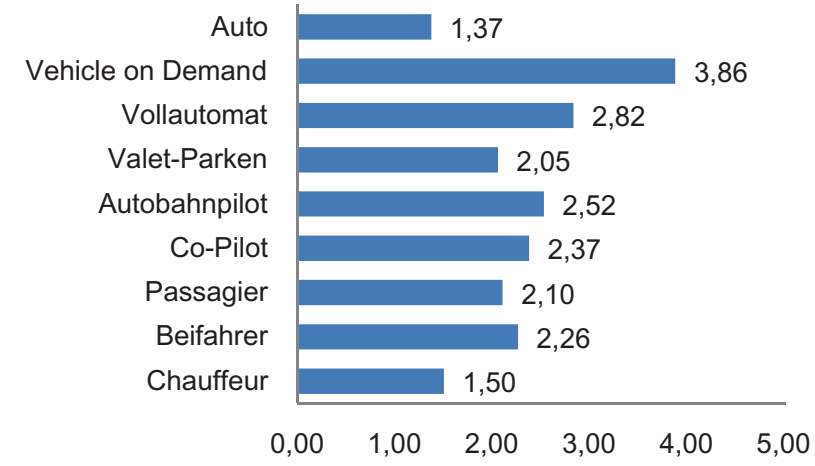


beregler $(1=$ Passagier und $10=$ Überwacher $)$ an, welche Rolle sie im autonomen Fahrzeug einnehmen wollen. Der arithmetische Mittelwert von 6,36 $(\mathrm{SD}=2,9)$ vermittelt eine Präferenz für die Rolle des aktiven Überwachers, dem fortwährend die Kontrolle des Fahrzeugs auf der Basis von stetig verfügbaren Systeminformationen ermöglicht wird. Die Rolle des passiven Passagiers ist auch auf affektiver Ebene $(d=2,1)$ noch sichtbar vom erwünschten Ideal $(d=0)$ entfernt.

\subsubsection{Kognitive und emotionale Repräsentationen der Nutzungsszenarien}

Wie oben beschrieben, wurde die Gesamtstichprobe in diesem Teil des Fragebogens zufallsbasiert in vier gleich große Untergruppen (jeweils $n=250$ ) aufgeteilt und einem der vier Nutzungsszenarien (autonome Fahrzeuge mit Autobahnpilot mit Verfügbarkeitsfahrer (1), mit Valet-Parken (2), vollautomatisierte Fahrzeuge mit Verfügbarkeitsfahrer (2) und Vehicle-on-Demand (4)) zugewiesen. Dies ermöglichte einen Intergruppenvergleich der Erwartungen und Einstellungen gegenüber den einzelnen Szenarien. Zu Beginn dieses Abschnitts wurden die Teilnehmer nach der Nutzungsbereitschaft der jeweils kurz beschriebenen Varianten des autonomen Fahrens gefragt. Autonome Fahrzeuge mit ValetParken finden die meiste Zustimmung (53 Prozent), gefolgt von vollautomatisierten Fahrzeugen mit Verfügbarkeitsfahrer (45 Prozent) und Fahrzeugen mit Autobahnpilot (42 Prozent). Die geringste Nutzungsabsicht wurde gegenüber dem Vehicle-on-Demand geäußert (35 Prozent). Die Unterschiede sind gemäß der durchgeführten einfaktoriellen Varianzanalyse (ANOVA) statistisch bedeutsam $(F(3,996)=4,528 ; p<.01)$. Der Bonferroni-Posthoc-Test (paarweiser Mittelwertvergleich) zeigt jedoch an, dass sich lediglich die UseCases Valet-Parken und Vehicle-on-Demand in der Nutzungsabsicht signifikant unterscheiden $(p<.01)$.

Auf die Frage, in welchem Umfang diverse Mobilitätsbedürfnisse durch die Nutzung eines autonomen Fahrzeugs erfüllt werden würden, zeigen sich teilweise unterschiedliche Einschätzungen im Vergleich der Szenarien. In Tab. 6.3 sind die Mittelwerte dieser Beurteilungen und statistische Resultate (ANOVA und Bonferroni-Post-hoc-Test) gegenübergestellt. In der Gesamtbetrachtung zeigt sich, dass autonome Fahrzeuge als komfortabel, stressfrei und umweltfreundlich wahrgenommen werden. Statistisch relevante Unterschiede im Vergleich der Use-Cases bestehen in puncto Stressfreiheit, Komfort, Sicherheit und Zeitersparnis. Valet-Parken adressiert dabei nach Einschätzung der Befragten die Bedürfnisse nach Zeitersparnis, Komfort, Stressfreiheit am effektivsten und erklärt somit die hohen Akzeptanzraten in dieser Variante autonomen Fahrens. Kritisch hervorzuheben sind die Sicherheitsbedenken beim Nutzungsszenario Vehicle-onDemand.

Die emotionale Bewertung der Use-Cases erfolgte hinsichtlich zehn unterschiedlicher Emotionen (Hoffnung, Gelassenheit, Zufriedenheit, Freude, Besorgnis, Ärger, Stress, Machtlosigkeit, Abneigung, Angst). Die Teilnehmer waren dabei aufgefordert anzugeben, welche Emotionen sie bei der antizipierten Nutzung der jeweiligen Variante autonomen Fahrens empfinden. Die Ergebnisse (s. Tab. 6.4) bestätigen die Tendenzen der oben dargestellten Unterschiede im Vergleich der Use-Cases. Mit dem Valet-Parken sind die stärks- 
Tab. 6.3 Arithmetisches Mittel $(M)$ und Standardabweichung $(S D)$ der Bedürfniserfüllung

\begin{tabular}{|l|l|l|l|l|l|}
\hline & $\begin{array}{l}\text { Auto- } \\
\text { bahnpilot }\end{array}$ & Valet-Parken & Vollautomat & $\begin{array}{l}\text { Vehicle- } \\
\text { on-Demand }\end{array}$ & \\
\hline Mobilitätsbedürfnis & $\mathrm{M}(\mathrm{SD})$ & $\mathrm{M}(\mathrm{SD})$ & $\mathrm{M}(\mathrm{SD})$ & $\mathrm{M}(\mathrm{SD})$ & $\mathrm{F}(3,996)$ \\
\hline Unabhängigkeit & $3,39(1,44)$ & $3,70_{4}(1,46)$ & $3,53(1,47)$ & $3,32_{2}(1,57)$ & $3,286^{*}$ \\
\hline Stressfreiheit & $3,72_{2}(1,55)$ & $4,13_{1,4}(1,43)$ & $3,93(1,50)$ & $3,67_{4}(1,79)$ & $4,509 * *$ \\
\hline Komfort & $3,78(1,42)$ & $4,12_{4}(1,39)$ & $4,06_{4}(1,38)$ & $3,63_{2,3}(1,58)$ & $6,364^{* *}$ \\
\hline Niedrige Kosten & $3,45(1,30)$ & $3,35(1,31)$ & $3,24(1,38)$ & $3,55(1,52)$ & 2,336 \\
\hline Umweltfreundlichkeit & $3,71(1,30)$ & $3,79(1,29)$ & $3,81(1,32)$ & $3,78(1,50)$ & 0,253 \\
\hline Sicherheit & $3,48(1,48)$ & $3,55(1,30)$ & $3,66_{4}(1,48)$ & $1,22_{3}(1,64)$ & $4,014^{* *}$ \\
\hline Soziales Ansehen & $2,86(1,39)$ & $2,87(1,35)$ & $2,97(1,38)$ & $2,87(1,49)$ & 0,354 \\
\hline Fahrerlebnis & $3,24(1,43)$ & $3,39_{4}(1,34)$ & $3,28(1,42)$ & $3,01_{4}(1,57)$ & $3,029 *$ \\
\hline Geringer Zeitaufwand & $3,40_{2}(1,37)$ & $4,00_{1,3,4}(1,40)$ & $3,43_{2}(1,39)$ & $3,36_{2}(1,52)$ & $11,534^{* *}$ \\
\hline Bering
\end{tabular}

Bemerkung: Die durch tiefer gestellte Zahlen markierten Mittelwerte weisen einen signifikanten Unterschied im Bonferroni-Post-hoc-Test (paarweiser Mittelwertvergleich) auf einem Niveau von $p=.05$ auf (z. B. tiefer gestellte 2 in der zweiten Zeile/ ersten Spalte indiziert einen signifikanten Unterschied zu dem entsprechenden Wert der zweiten Spalte)

${ }^{*} p<.05 ; * * p<.01$

ten positiven emotionalen Assoziationen verbunden. Die Gefühle Zufriedenheit, Gelassenheit und Freude sind signifikant stärker ausgeprägt als in den übrigen Szenarien. In den Szenarien Autobahnpilot, vollautomatisiertes Fahrzeug und Vehicle-on-Demand überwiegen die Empfindungen der Machtlosigkeit und der Angst. Das Gefühl, ausgeliefert zu sein, geht mit diesen Emotionen einher und stellt ein starkes Akzeptanzhindernis dar. Neben dem Valet-Parken evoziert lediglich das vollautomatisierte Fahrzeug überdurchschnittlich positive Emotionen wie Freude, Hoffnung und Zufriedenheit, obgleich die negativen Empfindungen in diesem Szenario überwiegen.

Diese Ergebnisse liefern ein differenziertes Bild der emotionalen Grundlagen, welche die wohl wichtigste Emotion im Kontext der Automatisierung konstituieren - das Vertrauen. Das Vertrauen in die beschriebenen Varianten des autonomen Fahrens wurde in dieser Befragung anhand von vier Items (z. B. „Ich kann mir vorstellen, mich in meiner Alltagsmobilität auf ein derartiges System zu verlassen") gemessen - analog zu den übrigen Einstellungsitems auf einer sechsstufigen Likert-Skala. Aus diesen Items wurde ein Summenindex gebildet. Wie nicht anders zu erwarten, ist das Vertrauen in Fahrzeuge mit ValetParken $(M=3,45 ; S D=1,31)$ am höchsten und gegenüber dem Vehicle-on-Demand am schwächsten ausgeprägt $(M=3,10 ; S D=1,42)$. Der Vertrauen in Fahrzeuge mit Autobahnpilot und in vollautomatisierte Fahrzeuge befindet sich auf annähernd gleichem Niveau $(M=3,36 ; S D=1,33$ zu $M=3,28 ; S D=1,33)$. Statistisch bedeutsam sind lediglich die Unterschiede zwischen dem Szenario Valet-Parken und Vehicle-on-Demand (BonferroniPost-hoc-Test, $\mathrm{p}<.05$ ). 
Tab. 6.4 Arithmetisches Mittel $(M)$ und Standardabweichung $(S D)$ der emotionalen Empfindungen

\begin{tabular}{|l|l|l|l|l|l|}
\hline & $\begin{array}{l}\text { Auto- } \\
\text { bahnpilot }\end{array}$ & Valet-Parken & Vollautomat & $\begin{array}{l}\text { Vehicle- } \\
\text { on-Demand }\end{array}$ & \\
\hline Emotion & $\mathrm{M}(\mathrm{SD})$ & $\mathrm{M}(\mathrm{SD})$ & $\mathrm{M}(\mathrm{SD})$ & $\mathrm{M}(\mathrm{SD})$ & $\mathrm{F}(3,996)$ \\
\hline Hoffnung & $3,04(1,30)$ & $3,16(1,35)$ & $3,23(1,32)$ & $3,00(1,39)$ & 1,504 \\
\hline Gelassenheit & $3,12(1,45)$ & $3,44_{4}(1,40)$ & $3,22(1,33)$ & $3,06_{2}(1,49)$ & $3,482^{* *}$ \\
\hline Zufriedenheit & $3,25(1,44)$ & $3,52_{4}(1,48)$ & $3,35(1,33)$ & $3,09_{2}(1,49)$ & $4,024 * *$ \\
\hline Freude & $3,07(1,44)$ & $3,43_{4}(1,42)$ & $3,30(1,33)$ & $3,06_{2}(1,44)$ & $4,135^{* *}$ \\
\hline Besorgnis & $3,50_{2}(1,47)$ & $2,97_{1,3,4}(1,49)$ & $3,54_{2}(1,43)$ & $3,52_{2}(1,63)$ & $8,474 * *$ \\
\hline Ärger & $2,66(1,34)$ & $2,49(1,37)$ & $2,75(1,34)$ & $2,79(1,45)$ & 2,371 \\
\hline Stress & $3,04_{2}(1,52)$ & $2,57_{1,3,4}(1,36)$ & $3,10_{2}(1,43)$ & $3,04_{2}(1,59)$ & $7,028^{* *}$ \\
\hline Machtlosigkeit & $3,72_{2}(1,59)$ & $3,04_{1,3,4}(1,51)$ & $3,63_{2}(1,45)$ & $3,82_{2}(1,67)$ & $12,770^{* *}$ \\
\hline Abneigung & $3,23_{2}(1,61)$ & $2,75_{1,3,4}(1,55)$ & $3,27_{2}(1,55)$ & $3,35_{2}(1,72)$ & $7,075^{* *}$ \\
\hline Angst & $3,39_{2}(1,57)$ & $2,68_{1,3,4}(1,40)$ & $3,24_{2}(1,43)$ & $3,37_{2}(1,65)$ & $12,075^{* *}$ \\
\hline
\end{tabular}

Bemerkung: Die durch tiefergestellte Zahlen markierten Mittelwerte weisen einen signifikanten Unterschied im Bonferroni-Post-hoc-Test (paarweiser Mittelwertvergleich) auf einem Niveau von $p=.05$ auf (z. B. tiefergestellte 4 in der zweiten Zeile/ zweiten Spalte indiziert einen signifikanten Unterschied zu dem entsprechenden Wert der vierten Spalte).

${ }^{*} p<.05 ; * * p<.01$

\subsubsection{Interventions-, Kontroll- und Erlebnisbedürfnisse}

Für die deutliche Mehrheit der befragten Personen (Autobahnpilot: 82 Prozent; Valet Parken: 81 Prozent; vollautomatisiertes Fahrzeug: 88 Prozent; Vehicle-on-Demand: 84 Prozent) ist die Möglichkeit, jederzeit die manuelle Kontrolle über das Fahrzeug wieder übernehmen bzw. den automatisierten Fahrvorgang abbrechen zu können, eines der zentralen Bedürfnisse. Gleichzeitig will nur eine Minderheit in den Szenarien mit Verfügbarkeitsfahrer (Autobahnpilot: 32 Prozent; vollautomatisiertes Fahrzeug: 48 Prozent) die Aufmerksamkeit nicht mehr auf den Verkehr richten und dem automatisierten System vollständig die Fahrzeugkontrolle überlassen. Dies spiegelt sich auch in dem für diese beiden UseCases mehrheitlich geäußerten Bedürfnis wider, die im Fahrzeug übliche Sitzposition während der automatisierten Fahrt nicht verlassen zu wollen (Autobahnpilot: 76 Prozent; vollautomatisiertes Fahrzeug: 79 Prozent). In allen vier Szenarien formuliert die überwiegende Anzahl der Teilnehmer den Wunsch, die Automation gemäß der eigenen Präferenzen hinsichtlich Fahrstil (z. B. komfortabel vs. sportlich) oder Streckenauswahl (z. B. zeiteffizient vs. umweltfreundlich) anpassen zu können (Autobahnpilot: 71 Prozent; Valet-Parken: 76 Prozent; vollautomatisiertes Fahrzeug: 72 Prozent; Vehicle-on-Demand: 82 Prozent).

Als wichtigster Vorteil der Nutzung autonomer Fahrzeuge wird die Möglichkeit erachtet, während der Fahrt die Landschaft genießen zu können (Autobahnpilot: 64 Prozent; vollautomatisiertes Fahrzeug: 72 Prozent; Vehicle-on-Demand: 72 Prozent; Valet-Parken: o. A.). Weiterhin wird die Option, sich ungestört mit den Fahrzeuginsassen unterhalten zu können, 
als sehr positiv wahrgenommen (Autobahnpilot: 63 Prozent; vollautomatisiertes Fahrzeug: 65 Prozent; Vehicle-on-Demand: 68 Prozent; Valet-Parken: o. A.). Erstaunlicherweise werden Tätigkeiten wie Internetsurfen (Autobahnpilot: 28 Prozent; vollautomatisiertes Fahrzeug: 39 Prozent; Vehicle-on-Demand: 46 Prozent; Valet-Parken: o. A.), Filme ansehen (Autobahnpilot: 23 Prozent; vollautomatisiertes Fahrzeug: 32 Prozent; Vehicle-on-Demand: 36 Prozent; Valet-Parken: o. A.), Arbeiten (Autobahnpilot: 22 Prozent; vollautomatisiertes Fahrzeug: 33 Prozent; Vehicle-on-Demand: 36,4 Prozent; Valet-Parken: o. A.) oder Entspannen bzw. Schlafen (Autobahnpilot: 31 Prozent; vollautomatisiertes Fahrzeug: 47 Prozent; Vehicle-on-Demand: 54 Prozent; Valet-Parken: o. A.) nur von einer Minderheit als positiver Aspekt des automatisierten Fahrens betrachtet. Als wichtigste Vorteile des Valet-Parkens werden die Entlastung bei der Parkplatzsuche (80 Prozent), die Sicherheit des Parkortes (78 Prozent), die entstehende frei verfügbare Zeit (76 Prozent) sowie die günstigeren Parkplatzoptionen außerhalb der Innenstädte wahrgenommen (76 Prozent).

\subsection{Zusammenfassung und Schlussfolgerung}

Die Interaktion zwischen Mensch und autonomem Fahrzeug stand im vorliegenden Beitrag im Fokus des Interesses. Vor dem Hintergrund der Annahme, dass automatisierte Fahrzeuge in absehbarer Zukunft immer noch auf die Verfügbarkeit und Kontrolle des Menschen angewiesen sein werden, wurden zunächst die kognitionspsychologischen Auswirkungen der Mensch-Maschine-Interaktion betrachtet. Im Weiteren wurde im Rahmen einer umfänglichen Onlinebefragung die Nutzerperspektive des autonomen Fahrens empirisch untersucht. Das besondere Augenmerk galt dabei den Einstellungen, Erwartungen und Emotionen - den mentalen Modellen - gegenüber dem Thema des autonomen Fahrens.

Aus den Forschungsergebnissen zu den psychologischen Folgen der Automatisierung in unterschiedlichen Domänen (z. B. Luftfahrt, Produktion) ist zu schließen, dass der Mensch auf dem Weg zum vollautomatisierten Fahrzeug noch stärker ins Zentrum der Aufmerksamkeit von Gestaltern und Entwicklern rücken muss. Bereits bei den heute verfügbaren teilautomatisierten Systemen zeigen sich bei Fahrern bekannte Probleme wie unangemessenes Vertrauen und reduziertes Situationsbewusstsein. Welche Auswirkungen höhere Automatisierungsgrade und die damit verbundene, länger währende mentale Entkoppelung langfristig auf die kognitiven und motorischen Fähigkeiten des Fahrers haben werden, ist noch weitestgehend unbekannt. Die diesbezüglich festgestellten Effekte bei hochtrainierten und erfahrenen Flugzeugpiloten sind jedoch alarmierend [38]. Training und die regelmäßige manuelle Ausführung automatisierbarer Fahrtätigkeiten scheinen somit ein wichtiges Instrument zu sein, um die benötigten und erwünschten Kompetenzen der Fahrer aufrechtzuhalten.

Solange der Mensch im Verfügbarkeitskonzept des automatisierten Fahrzeugs vorkommt - ob als Überwacher des Systems oder zur Übernahme der Fahraufgabe -, benötigen sowohl der Mensch als auch die Maschine eine geeignete Repräsentation des jeweils anderen Akteurs. Transparente und an das mentale System des Menschen angepasste Schnittstellen 
sind die Voraussetzung für das notwendige Situations- und Systembewusstsein im Umgang mit dem automatisierten Fahren. Andererseits muss auch das technische System in der Lage sein, den mentalen Zustand des Fahrers, seine Intentionen sowie sein Verhalten richtig zu interpretieren und in einem Fahrermodell dynamisch abzubilden. In adaptiven und kooperativen Gestaltungskonzepten werden diese Aspekte bereits in hochautomatisierten Fahrzeugen prototypisch umgesetzt $[44,67]$. Darüber hinaus arbeiten aktuell Automobilhersteller und Forschungseinrichtungen in diversen Projekten an den potenziellen Lösungen dieser Probleme (www.adaptive-ip.eu; www.incarin.de; www.urban-online.org).

In den Umfrageergebnissen werden teilweise vorhandene Widersprüche zwischen dem technisch Möglichen und den von der Bevölkerung gewünschten Innovationen sichtbar. Auch wenn man sich mehrheitlich an die Übernahme bestimmter Fahraufgaben (z. B. Geschwindigkeitsregelung) durch Assistenzsysteme gewöhnt hat, wollen die meisten buchstäblich nur ungern das Lenkrad aus der Hand geben. Die heutigen kognitiven und affektiven Repräsentationen zu der Rolle des Fahrers sind noch sehr stark mit dem traditionellen Bild des aktiven Chauffeurs verbunden. Die Vorstellung, im Fahrzeug die Rolle des passiven Passagiers einzunehmen, findet nur wenig Akzeptanz. Das traditionelle, manuell gesteuerte Auto ist in der Bevölkerung noch derart stark mit den Idealvorstellungen assoziiert, dass vollständig autonome Fahrzeuge mehrheitlich noch nicht den Mobilitätsbedürfnissen entsprechen. Die noch offene Frage ist, ob eine schrittweise, evolutionäre Automatisierung des Fahrzeugs die notwendigen Veränderungen mentaler Modelle bezüglich der Rollenerwartungen im autonomen Fahrzeug erreichen kann. Eine situationsspezifische Übergabe der Fahraufgaben an das autonome Fahrzeug kann dabei, wie an den hohen Akzeptanzraten des Valet-Parkens ersichtlich wird, eine mögliche zielführende Alternative darstellen.

Die Ergebnisse der Befragung liefern ferner Anhaltspunkte für mögliche Strategien dieser Transformation, die sich an den Bedürfnissen und Emotionen potenzieller Nutzer orientieren. Das Hauptargument bisheriger öffentlicher Debatten für die Einführung autonomer Fahrzeuge ist die erhöhte Sicherheit im Straßenverkehr. Diese Wahrnehmung wird jedoch nicht von der breiten Bevölkerung geteilt. Vielmehr sehen die Teilnehmer dieser Studie ihre Bedürfnisse wie Stressreduktion, Komfort und Umweltfreundlichkeit im Falle einer Nutzung autonomer Fahrzeuge erfüllt. Gleichwohl sind damit verbundene Emotionen wie Machtlosigkeit und Angst wirkungsmächtige, akzeptanzhemmende Faktoren. Der menschliche Denkapparat ist nicht in der Lage, das Risiko seltener Ereignisse objektiv einzuschätzen [58], sodass die Ängste und Bedenken zu irrationalen Entscheidungen führen können. Eine nutzerzentrierte Entwicklung bedeutet aus dieser Sicht, auf bestehende Bedürfnisse sowohl in der Kommunikation als auch in der konkreten Gestaltung der Systeme einzugehen.

Für den potenziellen Nutzer stellt sich letztendlich die Frage des Mehrwertes eines autonomen Fahrzeugs gegenüber dem bislang noch hoch in der Gunst stehenden manuell gesteuertem Vehikel. Worauf soll die Aufmerksamkeit gerichtet werden, wenn man sich schon nicht mehr aus Sicherheitsgründen mit der Fahrzeugsteuerung beschäftigen „darf"? Entgegen den Erwartungen haben die Teilnehmer mehrheitlich nicht das erweiterte Infotainment-Sortiment von Internet bis Fernsehen vor Augen, sondern wollen vielmehr ungestört 
den Blick auf die Landschaft genießen. Wie stabil und valide diese Aussagen im tatsächlichen Umgang mit automatisierten Fahrzeugen sind, werden zukünftige Studien zeigen müssen. Vielleicht steht dieses Bedürfnis aber auch in der Tradition deutscher Romantik und bietet einen neuen Impuls für die Gestaltung des automatisierten, „naturnahen“ Raums.

\section{Literatur}

1. Wilson, J.R., Rutherford, A.: Mental Models: Theory and Application in Human Factors. Hum. Factors J. Hum. Factors Ergon. Soc. 31, 617-634 (1989)

2. Bainbridge, L.: Ironies of automation. In: Johannsen, G. (ed.) Analysis, design and evaluation of man-machine systems. pp. 151-157. Pergamon (1982)

3. Norman, D.: The "problem" with automation: inappropriate feedback and interaction, not "over-automation.”Philos. Trans. R. Soc. B Biol. Sci. 327, 585-593 (1990)

4. Sheridan, T.B.: Supervisory control. In: Salvendy, G. (ed.) Handbook of human factors. pp. 1243-1268. Wiley, New York (1987)

5. Boeing Commercial Airline Group: Statistical summary of commercial jet aircraft accidents: Worldwide operations 1959-2005., http://www.boeing.com/news/techissues/pdf/statsum.pdf

6. Dismukes, R.K., Berman, B.A., Loukopoulos, L.D.: The Limits of Expertise: Rethinking Pilot Error and the Causes of Airline Accidents. Ashgate Publishing, Ltd. Federal (2007)

7. Bainbridge, L.: Ironies of Automation. Automatica. 19, 775-779 (1983)

8. Endsley, M.R., Kiris, E.O.: The Out-of-the-Loop Performance Problem and Level of Control in Automation. Hum. Factors J. Hum. Factors Ergon. Soc. 37, 381-394 (1995)

9. Madhavan, P., Wiegmann, D.A.: Similarities and differences between human - human and human - automation trust: an integrative review. Theor. Issues Ergon. Sci. 8, 277-301 (2007)

10. Onnasch, L., Wickens, C.D., Li, H., Manzey, D.: Human Performance Consequences of Stages and Levels of Automation: An Integrated Meta-Analysis. Hum. Factors J. Hum. Factors Ergon. Soc. 56, 476-488 (2013)

11. Endsley, M.R.: Situation awareness. In: Salvendy, G. (ed.) Handbook of human factors and ergonomics. pp. 528-542. Wiley, New York (2006)

12. Endsley, M.R., Bolte, B., Jones, D.G.: Designing for situation awareness: An approach to human-centered design. Taylor \& Francis, London (2003)

13. Billings, C.E.: Aviation Automation: The Search for a Human-Centered Approach. Human Factors in Transportation. Lawrence Erlbaum Associates Publishers (1997)

14. Parasuraman, R., Sheridan, T.B., Wickens, C.D.: A model for types and levels of human interaction with automation. Syst. Man Cybern. Part A Syst. Humans, IEEE Trans. 30, 286-297 (2000)

15. Christoffersen, K., Woods, D.D.: How to make automated systems team players. In: Salas, E. (ed.) Advances in Human Performance and Cognitive Engineering Research. pp. 1-12. Elsevier Science Ltd. (2002)

16. Kaber, D.B., Riley, J.M., Tan, K.-W., Endsley, M.R.: On the Design of Adaptive Automation for Complex Systems. Int. J. Cogn. Ergon. 5, 37-57 (2001)

17. Inagaki, T.: Traffic systems as joint cognitive systems: issues to be solved for realizing human-technology coagency. Cogn. Technol. Work. 12, 153-162 (2010)

18. Prevot, T., Homola, J.R., Martin, L.H., Mercer, J.S., Cabrall, C.D.: Toward Automated Air Traffic Control - Investigating a Fundamental Paradigm Shift in Human/Systems Interaction. Int. J. Hum. Comput. Interact. 28, 77-98 (2012)

19. Challenger, R., Clegg, C.W., Shepherd, C.: Function allocation in complex systems: reframing an old problem. Ergonomics. 56, 1051-69 (2013) 
20. Grote, G., Weyer, J., Stanton, N.A.: Beyond human-centred automation - concepts for humanmachine interaction in multi-layered networks. Ergonomics. 57, 289-94 (2014)

21. Hancock, P.A.: Automation: how much is too much? Ergonomics. 57, 449-54 (2014)

22. Arthur, C.: Google's driverless car: no steering wheel, two seats, $25 \mathrm{mph}$, http://www.theguardian.com/technology/2014/may/28/google-reveals-driverless-car-prototype

23. Cummings, P.M.L., Ryan, J.: Shared Authority Concerns in Automated Driving Applications. http://web.mit.edu/aeroastro/labs/halab/papers/cummingsryan_driverless2013_draft.pdf. Letzter Zugriff: 28.08.2014

24. Merat, N., Lee, J.D.: Preface to the Special Section on Human Factors and Automation in Vehicles: Designing Highly Automated Vehicles With the Driver in Mind. Hum. Factors J. Hum. Factors Ergon. Soc. 54, 681-686 (2012)

25. Hoogendoorn, R., Arem, B. Van, Hoogendoorn, S.: Automated Driving, Traffic Flow Efficiency And Human Factors: A Literature Review. Transportation Research Board 93rd Annual Meeting. pp. 1-18. Transportation Research Board (2014)

26. Lee, J.D., See, K.A.: Trust in Automation: Designing for Appropriate Reliance. Hum. Factors J. Hum. Factors Ergon. Soc. 46, 50-80 (2004)

27. Moray, N., Inagaki, T.: Attention and complacency. Theor. Issues Ergon. Sci. 1, 354-365 (2000)

28. Ghazizadeh, M., Peng, Y., Lee, J.D., Boyle, L.N.: Augmenting the Technology Acceptance Model with Trust: Commercial Drivers'Attitudes towards Monitoring and Feedback. Proc. Hum. Factors Ergon. Soc. Annu. Meet. 56, 2286-2290 (2012)

29. Kazi, T., Stanton, N., Walker, G., Young, M.: Designer driving: drivers' conceptual models and level of trust in adaptive cruise control. Int. J. Veh. Des. 45, 339-360 (2007)

30. Koustanai, A., Cavallo, V., Delhomme, P., Mas, A.: Simulator Training With a Forward Collision Warning System: Effects on Driver-System Interactions and Driver Trust. Hum. Factors J. Hum. Factors Ergon. Soc. 54, 709-721 (2012)

31. Stanton, N.A., Young, M.S.: Driver behaviour with adaptive cruise control. Ergonomics. 48, 1294-313 (2005)

32. Rajaonah, B., Tricot, N., Anceaux, F., Millot, P.: The role of intervening variables in driver-ACC cooperation. Int. J. Hum. Comput. Stud. 66, 185-197 (2008)

33. Flemisch, F., Kelsch, J., Löper, C., Schieben, A., Schindler, J.: Automation spectrum, inner/outer compatibility and other potentially useful human factors concepts for assistance and automation. In: Waard, D. de, Flemisch, F., Lorenz, B., Oberheid, H., and Brookhuis, and K. (eds.) Human Factors for Assistance and Automation. pp. 1-16. Shaker, Maastricht (2008).

34. Beggiato, M., Krems, J.F.: The evolution of mental model, trust and acceptance of adaptive cruise control in relation to initial information. Transp. Res. Part F Traffic Psychol. Behav. 18, 47-57 (2013)

35. Verberne, F.M.F., Ham, J., Midden, C.J.H.: Trust in Smart Systems: Sharing Driving Goals and Giving Information to Increase Trustworthiness and Acceptability of Smart Systems in Cars. Hum. Factors J. Hum. Factors Ergon. Soc. 54, 799-810 (2012)

36. Waytz, A., Heafner, J., Epley, N.: The Mind in the Machine: Anthropomorphism Increases Trust in an Autonomous Vehicle. J. Exp. Soc. Psychol. 52, 113-117 (2014)

37. Lee, J.D., Moray, N.: Trust, self-confidence, and operators' adaption to automation. Int. J. Human-Computer Stud. 40, 152-184 (1994)

38. Federal Aviation Administration: Safety Alert for Operators 13002. F. S. Service. Departement of Transportation, Washington DC (2013)

39. Buld, S., Krüger, H.-P., Hoffmann, S., Kaussner, A., Tietze, H., Totzke, I.: Wirkungen von Assistenz und Automation auf Fahrerzustand und Fahrsicherheit. Veröffentlichter Abschlussbericht Projekt EMPHASIS: Effort- Management und Performance-Handling in sicherheitsrelevanten Situationen. Würzburg: Interdisziplinäres Zentrum für Verkehrswissenschaften an der Universität Würzburg (IZVW), Würzburg: (2002) 
40. Ward, N.J.: Task automation and skill development in a simplified driving task. In Proceedings of the XIVth Triennial Congress of the International Ergonomics Association and 44th Annual Meeting of the Human Factors and Ergonomics Society. pp. 302-305, San Diego, CA (Santa Monica, CA: HFES) (2000)

41. Ma, R., Kaber, D.B.: Situation awareness and workload in driving while using adaptive cruise control and a cell phone. Int. J. Ind. Ergon. 35, 939-953 (2005)

42. Merat, N., Jamson, A.H., Lai, F.C.H., Carsten, O.: Highly Automated Driving, Secondary Task Performance, and Driver State. Hum. Factors J. Hum. Factors Ergon. Soc. 54, 762-771 (2012).

43. Gasser, T.M., Arzt, C., Ayoubi, M., Bartels, A., Eier, J., Flemisch, F., Häcker, D., Hesse, T., Huber, W., Lotz, C., Maurer, M., Ruth-Schumacher, S., Schwarz, J., Vogt, W.: BASt-Bericht F 83: Rechtsfolgen zunehmender Fahrzeugautomatisierung. Bremerhaven. (2012)

44. Flemisch, F.O., Bengler, K., Bubb, H., Winner, H., Bruder, R.: Towards cooperative guidance and control of highly automated vehicles: H-Mode and Conduct-by-Wire., http://www.ncbi.nlm. nih.gov/pubmed/24559139, (2014)

45. Craik, K.J.W.: The nature of explanation. Cambridge University Press, Cambridge, England (1943)

46. Johnson-Lairds, P.N.: Mental Models: Towards a Cognitive Science of Language, Influence and Consciousness. Cambridge University Press, Cambridge, UK (1983)

47. Jones, N.A., Ross, H., Lynam, T., Perez, P., Leitch, A.: Mental Models: An Interdisciplinary Synthesis of Theory and Methods. Ecol. Soc. 16, 46 (2011)

48. Moray, N.: Models of models of - mental models. In: Moray, N. (ed.) Ergonomics: major writings. pp. 506-552. Taylor and Francis, London, UK. (2004)

49. Nersessian, N.J.: The cognitive basis of model-based reasoning in science. In: Carruthers, S.S. and Siegal, M. (eds.) The cognitive basis of science. pp. 133-153. Cambridge University Press, Cambridge, UK (2002)

50. Norman, D.A.: Some observations on mental models. In: Baecker, R.M. and Buxton, W.A.S. (eds.) Human-computer Interaction. pp. 241-244. Morgan Kaufmann Publishers Inc., San Francisco, CA, USA (1987)

51. Zhang, W., Xu, P.: Do I have to learn something new? Mental models and the acceptance of replacement technologies. Behav. Inf. Technol. 30, 201-211 (2011)

52. d'Apollonia, S.T., Charles, E.S., Boyd, G.M.: Acquisition of Complex Systemic Thinking: Mental Models of Evolution. Educ. Res. Eval. 10, 499-521 (2004)

53. Gefen, D., Karahanna, E., Straub, D.W.: Inexperience and experience with online stores: The importance of tam and trust. IEEE Trans. Eng. Manag. 50, 307-321 (2003)

54. Stanton, N., Young, M.S.: A proposed psychological model of driving automation. Theor. Issues Ergon. Sci. 1, 315-331 (2000)

55. Boer, E.R., Hoedemaeker, M.: Modeling driver behavior with different degrees of automation: A Hierarchical Decision Framework of Interacting Mental Models. In: Al., A.G.G. et (ed.) 17th European Annual Conference on Human Decision Making and Manual Control. pp. 63-72. Elsevier, Amsterdam (1989)

56. Schröder, T., Huck, J., de Haan, G.: Transfer sozialer Innovationen: Eine zukunftsorientierte Fallstudie zur nachhaltigen Siedlungsentwicklung. VS Verlag fuer Sozialwissenschaften, Wiesbaden (2011)

57. Gigerenzer, G., Goldstein, D.G.: Reasoning the fast and frugal way: models of bounded rationality. Psychol. Rev. 103, 650-69 (1996)

58. Kahnemann, D.: Thinking, fast and slow. Penguin, New York (2011)

59. Loewenstein, G.F., Lerner, J.S.: The role of affectt in decision making. In: Davidson, R., Scherer, K., and Goldsmith, H. (eds.) Handbook of affectivce science. pp. 619-642. Oxford University Press, New York (2003)

60. Kunda, Z.: The case for motivated reasoning. Psychol. Bull. 108, 480-498 (1990) 
61. MacKinnon, N.J., Heise, D.R.: Self, Identity, and Social Institutions. NY: Palgrave Macmillan, New York (2010)

62. Wolf, I., Schröder, T., Neumann, J., de Haan, G.: Changing minds about electric cars: An empirically grounded agent-based modeling approach. Vorabdruck: http://arxiv.org/abs/1405.6230, (2014)

63. Continental AG: Continental-Mobilitätsstudie 2013. (2013)

64. Infas, DLR: Mobilität in Deutschland (MiD) 2008. Infas Institut für angewandte Sozialwissenschaft GmbH, Deutsches Zentrum für Luft- und Raumfahrt e. V. (DLR), Bonn, Berlin (2008)

65. Osgood, C.E., Suci, G.J., Tannenbaum, P.H.: The Measurement of Meaning. Linguistic Society of America, Urbana (1957)

66. Heise, D.R.: Expressive order: Confirming sentiments in social action. Springer, New York (2007)

67. Löper, C., Kelsch, J., Flemisch, F.: Kooperative, manöverbasierte Automation und Arbitrierung als Bausteine für hochautomatisiertes Fahren. In: Gesamtzentrum für Verkehr Braunschweig (ed.) Automatisierungs-, Assistenzsysteme und eingebette Systeme für Transportmittel. pp. 215-237, Braunschweig (2008)

68. Buld, S., Tietze, H., Krüger, H.: Auswirkungen von Teilautomation auf das Fahren. In: Maurer, M.; Stiller, C. (Hrsg.): Fahrerassistenzsysteme mit maschineller Wahrnehmung. S. 161-187, Springer, Berlin (2005) 